\title{
Philonsorbonne
}

14 | 2020

Année 2019-2020

\section{Le fou, le solipsiste et le philosophe}

\section{Valérie AUCOUTURIER}

\section{(2) OpenEdition}

\section{Journals}

Édition électronique

URL : https://journals.openedition.org/philonsorbonne/1511

DOI : 10.4000/philonsorbonne.1511

ISSN : 2270-7336

\section{Éditeur}

Publications de la Sorbonne

\section{Édition imprimée}

Date de publication : 14 février 2020

Pagination : 161-177

ISSN : 1255-183X

\section{Référence électronique}

Valérie AUCOUTURIER, « Le fou, le solipsiste et le philosophe », Philonsorbonne [En ligne], 14 | 2020

mis en ligne le 03 mars 2020, consulté le 08 juin 2021. URL : http://journals.openedition.org/

philonsorbonne/1511 ; DOI : https://doi.org/10.4000/philonsorbonne.1511 


\title{
Le fou, le solipsiste et le philosophe
}

\author{
Valérie AUCOUTURIER \\ (Université Saint-Louis - Bruxelles) \\ (Centre Prospéro. Langage, Image, Connaissance)
}

On trouve parfois, chez Wittgenstein, la figure du fou associée à celle du philosophe. Mais il s'agit alors généralement du philosophe au sens péjoratif du terme. C'est-à-dire au sens de celui qui profère des non-sens, qui emploie des expressions décrochées de tout usage, sans se donner les moyens de leur conférer un sens. C'est ce dont témoignent ces extraits de De la certitude :

467. - Je suis assis avec un philosophe dans le jardin ; il dit à maintes reprises : «je sais que ceci est un arbre» tout en désignant un arbre près de nous. Une tierce personne arrive et entend cela, et je lui dis : «Cet homme n'est pas fou. Nous faisons de la philosophie».

468. - Quelqu'un dit hors de propos : « Ceci est un arbre». [...] Sur ce, je lui demande: "Qu'entendais-tu par-là? », et il répond: "C'était une information qui t'était adressée ». N'aurais-je alors pas tout lieu de penser qu'il ne sait pas ce qu'il dit s'il est assez fou pour vouloir me communiquer une telle information ? ${ }^{1}$

Est ici mise en scène la façon dont la parole du philosophe apparaît décrochée de la parole ordinaire, comme si ce dernier ressentait le besoin étrange de dire ou d'affirmer des choses qui précisément vont ordinairement sans dire. C'est d'ailleurs pourquoi il est curieux de les dire. Si l'on conçoit aisément la portée critique de ces remarques et de la comparaison peu flatteuse du fou et du philosophe, on peut aussi y voir une manière de situer la parole philosophique par rapport à celle du fou, voire même une manière de l'autoriser dans une certaine mesure. En effet, dire « Cet homme n'est pas

1. L. Wittgenstein, De la certitude, trad. D. Moyal-Sharrock, Paris, Gallimard, 2006. 
fou. Nous faisons de la philosophie », n'est-ce pas dire que, s'il n'était pas en train de philosopher, sans doute serait-il fou, mais qu'il est précisément en train de philosopher, et donc qu'il n'est pas fou ? «Je sais que ceci est un arbre » n'est pas ici prononcé dans un moment de délire, mais dans le contexte d'une conversation philosophique. Un contexte dans lequel le philosophe voudrait dire quelque chose en prononçant ces mots, comme par exemple énoncer une certitude, donner un exemple d'une chose que nous "savons» précisément parce qu'elle se présente comme une évidence, comme ce qui va sans dire.

Le rapprochement contrasté que propose Wittgenstein entre le philosophe et la figure du fou possède ainsi une double portée critique. Il suggère, d'une part, que la parole philosophique, lorsqu'elle prétend s'émanciper de l'attitude ordinaire, s'approche dangereusement de la parole délirante et insensée du fou. Mais il suggère, d'autre part, que la parole philosophique n'est pas tout à fait « hors de propos » comme l'est celle du fou. Du moins pas de la même façon.

Ainsi, lorsque René Descartes, au début des Méditations métaphysiques $^{2}$, se propose de mettre entre parenthèses l'existence du monde extérieur, il philosophe. De même lorsque le Husserl des Méditations cartésiennes $^{3}$ poursuit et radicalise le geste cartésien dans l'épochè phénoménologique. Mais celui qui voudrait étendre l'attitude philosophique (en l'occurrence le doute méthodique) au contexte ordinaire, quotidien, passerait pour fou, ou du moins très étrange. Dès lors, que peut bien avoir de fou le fait de répéter, en désignant l'arbre en question : " Je sais que ceci est un arbre », ou de dire inopinément « Ceci est un arbre » en montrant un arbre devant soi ? On peut bien sûr imaginer des circonstances dans lesquelles ceci n'aurait rien de fou. Par exemple, dans un contexte où je devrais distinguer des arbres réels d'arbres holographiques, ou dans un contexte où j'apprendrais à distinguer, en botanique, les arbres de plantes herbacées qui leurs ressemblent mais n'en sont pas, comme le bananier; ou encore, dans un contexte où il serait question d'apprendre une langue ou d'apprendre à parler. Ce qui semble fou dans la parole du philosophe, c'est qu'elle est censée lever la possibilité d'un doute non pas seulement improbable, mais qui n'a pas lieu d'être. Elle est à ce titre littéralement « hors de propos ».

Ainsi la figure du philosophe ressemble-t-elle à celle du fou lorsque le premier prend des remarques conceptuelles, touchant aux limites du sens, pour des remarques empiriques, touchant aux possibilités des phénomènes. Autrement dit, si, dans l'exemple inventé par Wittgenstein, la parole du philosophe pourrait avoir un sens, ce serait seulement dans la mesure où elle ne prétendrait pas lever un pseudo-doute (élevé au rang de possibilité empirique), mais seulement fournir un exemple de ce qui précisément va sans dire - de «certitude » (de ce qui, d'un point de vue logique, exclut

2. R. Descartes, Méditations métaphysiques, Paris, Le livre de poche, 1990.

3. E. Husserl, Méditations cartésiennes, Paris, PUF, 1994. 
justement le doute). Une certitude qui ne peut précisément pas tenir le rôle conjurateur de fondement épistémologique que voudrait lui faire tenir le philosophe sceptique. Précisément pas, parce que son caractère de certitude tient à ce qu'elle va sans dire. L'énoncer, c'est suggérer à tort qu'on pourrait en douter. C'est chercher à lui donner un rôle magique qu'elle n'a pas : celui de socle de la connaissance. Voilà pourquoi la parole du philosophe peut nous sembler folle.

La parole du philosophe a ceci de fou qu'elle se permet d'étendre le doute là où le doute n'est pas simplement impossible - en raison d'une évidence factuelle - mais là où le concept de doute lui-même est inopérant en raison d'une impossibilité logique. Ainsi, bien qu'il existe des situations concrètes dans lesquelles nous concevons qu'un tel doute serait légitime, nous ne pouvons pas commencer par apprendre à douter que nous avons deux mains, que les objets (par exemple les arbres) disparaissent lorsqu'ils sont hors de notre champ perceptuel, etc., pour ensuite conjurer ce doute au moyen d'une connaissance solide qui viendrait se superposer à la croyance naïve. Au contraire, le doute n'intervient que sur fond de stabilité ; le doute ne peut émerger que de conditions épistémiques exceptionnelles (ce qui n'a pas nécessairement à voir avec leur fréquence) et secondaires dans l'ordre de l'apprentissage. Bref, le doute généralisé, le doute de principe, ne peut pas être le doute du quotidien ou de l'ordinaire. Il est nécessairement celui du philosophe et, s'il devient quotidien, celui du fou.

En partant de cette analogie wittgensteinienne entre la figure du fou et celle du philosophe, j'aimerais, dans cet article, explorer la question de savoir dans quelle mesure nous pouvons donner sens à un discours délirant. Pour cela, je partirai de deux analyses philosophiques de la schizophrénie ${ }^{4}$, desquelles je me départirai ensuite. J'ajoute, par précaution, que ces approches sur lesquelles je m'appuie ne prétendent pas offrir une analyse exhaustive du diagnostic de schizophrénie, mais s'appuient, pour l'une - il s'agit de l'analyse de Louis Sass dans Les Paradoxes du délire $e^{5}$ - sur l'expérience clinique, et pour l'autre - il s'agit des analyses de collègues psychologues, philosophes et informaticiens de l'université de Lorraine (Manuel Rebuschi, Maxime Amblard et Michel Musiol) - sur des analyses de conversations avec des patients étiquetés "schizophrènes ", dans des institutions psychiatriques françaises ${ }^{6}$.

Ces deux approches ont en effet en commun de chercher à rendre compte de manière rationnelle - c'est-à-dire intelligible pour celui qui

4. Sachant que je n'ai pas d'expérience clinique directe de la schizophrénie et que je dois faire confiance sur ce point aux auteurs sur lesquels je m'appuie. J'insiste d'ailleurs sur le rôle avant tout heuristique de la schizophrénie pour l'analyse que je propose, qui vise davantage la question philosophique des limites du sens que le problème de la maladie mentale. Libre à chacun de voir dans ces modestes analyses la possibilité d'un éclairage dudit problème.

5. L. A. Sass, Les Paradoxes du délire, trad. P.-H. Castel, Paris, Ithaque, 2010.

6. M. Rebuschi, M. Amblard, M. Musiol, « Schizophrénie, logicité et perspective en première personne », L'évolution psychiatrique, vol. 78, $\mathrm{n}^{\circ}$ 1, 2013, p. 127-141. 
n'est pas dans la peau du schizophrène - du point de vue de la pathologie. Autrement dit, il s'agit de rendre raison du délire en montrant, pour parler comme Wittgenstein, ce qu'il a de commun avec la forme de vie de celui qui ne le vit pas. Pour ce faire, Sass s'appuie largement sur une comparaison entre le schizophrène et la figure wittgensteinienne paradoxale du solipsiste, tandis que Rebuschi et al. s'appuient, entre autres, sur l'analyse quinienne du principe de charité 7 .

Pour fonctionner sur la base d'une communauté de forme de vie, ces analyses doivent donc laisser de côté, au moins pour un temps, la difficile question de la pertinence de la distinction entre "nous » (sujets ordinaires ou usagers ordinaires du langage) et «eux» (sujets extraordinaires ou schizophrènes ${ }^{8}$ ); ainsi que celle de la validité théorique (du point de vue des catégories) et empirique (du point de vue de l'étiquetage individuel des patients) du diagnostic de schizophrénie. Mais, du point de vue conceptuel, ces analyses nous permettent néanmoins d'enrichir la réflexion sur d'autres types de distinctions entre sujets ordinaires et extraordinaires: génies, psychopathes, terroristes, nazis, etc., et d'aborder la question de savoir dans quelle mesure ces formes de vie extraordinaires ne sont pas complètement étrangères à la nôtre - dans quelle mesure on ne sort pas de l'humain, comme ce serait le cas avec le lion de Wittgenstein lorsqu'il remarque : « Si un lion pouvait parler nous ne le comprendrions pas $»{ }^{9}$.

Je voudrais donc explorer cette idée, défendue par Rebuschi et al. ${ }^{10}$, suivant laquelle le discours schizophrénique n'est pas à proprement parler irrationnel, car nous pouvons rendre compte de sa rationalité depuis ce qu'ils nomment un point de vue en première personne. Ainsi, une large partie de cet article sera consacrée à la question de savoir comment comprendre ici l'idée d'un point de vue en première personne. Pour Rebuschi et al., la perspective en première personne se distingue, sans l'exclure, de la perspective en troisième personne, qu'ils assimilent à toute perspective qu'ils qualifient de "réductionniste »- de type, par exemple, neurobiologique ou même psychanalytique (compris comme une explication par une causalité inconsciente). La perspective en première personne consiste, selon eux, en une prise en compte du point de vue du schizophrène, et en particularité de sa rationalité, à partir du principe de charité, hérité de Quine et de Davidson ${ }^{11}$. Leur perspective s'inspire également, tout en s'en distinguant, de l'analyse par Louis Sass de ce qu'il appelle le «solipsisme

7. W.V.O. Quine, Le Mot et la chose, trad. J. Dopp et al., Paris, Flammarion, 2010, ch. II, $\S 13$.

8. Dont nous devons bien reconnaître qu'ils emploient aussi le langage ordinaire.

9. L. Wittgenstein, Recherches philosophiques, trad. F. Dastur et al., Paris, Gallimard, 2005, II, xi.

10. M. Rebuschi, M. Amblard, M. Musiol, « Schizophrénie logicité et perspective en première personne », art. cit.

11. D. Davidson, «On the Very Idea of a Conceptual Scheme », Inquiries into Truth and Interpretation, Oxford, Clarendon Press, 1974, ch. 13. 
schizophrénique » (s'inspirant ici de la figure philosophique du solipsiste, largement empruntée à Wittgenstein).

Dans ses grandes lignes, le principe de charité invoqué consiste dans une analyse de notre rapport aux attitudes et aux discours d'autrui et affirme que nous accordons (ou devons accorder) à ces attitudes et discours (aussi étranges puissent-ils être) une logicité et une rationalité maximales. Ce principe est constitué, notamment par Quine, à partir de la figure de l'anthropologue confronté à une langue ou des pratiques inconnues de lui.

L'intérêt d'une telle perspective anthropologique sur la pathologie réside dans sa capacité à ne pas exclure par principe la pathologie, si ce n'est du domaine de la rationalité, du moins du domaine du compréhensible ou du saisissable, c'est-à-dire d'une commune humanité. Même si l'humanité ou la forme de vie ne sont pas forcément les plus petits dénominateurs communs, ou plutôt se révèlent insatisfaisants comme caractérisation conceptuelle d'un plus petit dénominateur commun, qui demeure sans doute introuvable comme tel, si l'on veut en donner une expression positive. Ce qui compte dans ces approches c'est qu'il y a du commun, du commensurable; notamment en raison d'une commune forme de vie, mais pas seulement; notamment en raison d'un langage commun, mais pas seulement; notamment en raison d'un environnement commun, etc. Ce qui compte également, et qui fait que la question d'une communication et d'une compréhension possible se pose, c'est qu'il y a du non-commun, quelque chose qui nous échappe, qui empêche de pouvoir se mettre totalement à la place d'autrui.

C'est d'ailleurs la raison pour laquelle la tentative par Sass de ressaisir le point de vue du schizophrène à travers la figure du solipsiste est particulièrement intéressante. Ses analyses s'appuient en partie, mais pas exclusivement, sur les mémoires de Schreber (analysées par Freud ${ }^{12}$ ). À partir des analyses wittgensteiniennes de la figure du solipsiste, Sass défend l'idée que le schizophrène ne souffre pas d'un «défaut d'épreuve de réalité », comme le soutient la thèse standard, mais d'une forme d'hyperréflexivité ${ }^{13}$. En d'autres termes, ce n'est pas que le schizophrène ne parviendrait pas à distinguer son délire de la réalité, mais plutôt que la réalité aurait pour lui un caractère irréel. Sass compare ainsi l'attitude du schizophrène à l'attitude du philosophe moderne (cartésien et post-cartésien) qui met en cause l'existence du monde extérieur ou sa propre existence en tant que sujet, voire, comme Kant, les conditions de possibilités mêmes de la connaissance. Le schizophrène est comme prisonnier d'un théâtre postcartésien et post-kantien ${ }^{14}$.

12. Voir S. Freud, «Le Président Schreber. Remarques psychanalytiques sur un cas de paranoïa », in Cinq psychanalyses, Paris, PUF, 1935.

13. L. Sass, Les Paradoxes du délire, op. cit., p. 137.

14. Ibid., p. 122. 
L'intérêt de cette comparaison entre le fou, le solipsiste et le philosophe est double. Elle permet, d'une part, dans une certaine mesure, de rendre raison du commun entre l'ordinaire et ces trois figures. Autrement dit, elle permet de ne pas cantonner le schizophrène au domaine de l'inexpressible ou de l'incommunicable; de ne pas faire du fou une autre figure du lion de Wittgenstein. Mais ceci nécessite, on va le voir, de tenir ensemble deux dimensions paradoxales du solipsisme. Elle permet, d'autre part, si l'on suit Rebuschi et al., de comprendre les altérations de la communication avec un schizophrène, en empruntant à Sass l'idée que ce dernier ne parle pas depuis un point de vue ordinaire, mais depuis celui d'une épochè.

Trois difficultés au moins me semblent cependant émerger de cette perspective. La première est relative au principe de charité (quel que soit son degré d'application) qui s'appuie sur une forme de subjectivisme méthodologique suivant lequel c'est seulement par inférence, à partir de mes propres expériences ou de mon propre point de vue, ou par interprétation, que j'accède à la vie mentale d'autrui. La deuxième difficulté porte sur ce qu'il faudrait comprendre par l'adoption d'un point de vue en première personne: qu'est-ce que cela signifie, si ce n'est être littéralement la personne concernée ? Ce qui conduit à soulever la troisième difficulté : puisqu'il est impossible d'être littéralement la personne concernée, en quoi l'adoption méthodologique d'un point de vue en première personne ne revient-elle pas simplement à transposer «ma» propre rationalité ou «notre» rationalité à l'autre. Je ne traiterai pas ici ces trois difficultés systématiquement, mais je vais tâcher de les lier à une difficulté centrale dans la compréhension du solipsisme et du privé chez Wittgenstein.

Dans ce qui suit, je commencerai donc par présenter l'interprétation que Rebuschi et ses collègues proposent du principe de charité et de son application à la figure du solipsiste. Je m'interrogerai ensuite sur la question de la rationalité du discours schizophrène à travers un examen de la façon dont il faut comprendre la thèse de rationalité du locuteur appliquée au solipsiste. Je proposerai enfin pour conclure une approche critique de la notion de déficit interactionnel dans la parole du schizophrène, compris comme déficit qui engage un simple contexte linguistique.

\section{Principe de charité et solipsisme}

Le projet de formalisation des conversations avec des patients schizophrènes mené par Rebuschi et al. se fonde sur une certaine application du principe de charité, dont ils relèvent trois compréhensions possibles :

1. la thèse de logicité forte suivant laquelle on considère que le locuteur se conforme à la logique classique ;

2. la thèse de logicité faible suivant laquelle on considère que le locuteur respecte le principe de non-contradiction (et n'admet pas $\mathrm{p}$ et non-p simultanément) ; 
3. la thèse de rationalité suivant laquelle on considère que le locuteur est rationnel (même s'il ne se conforme pas au principe de non-contradiction).

Sur la base d'analyses formelles de conversations avec des schizophrènes, Rebuschi et al. affirment qu'en dépit de contradictions apparentes, si l'on se place du point de vue du patient schizophrène, on peut défendre non seulement la thèse de rationalité, mais aussi la thèse de logicité forte. En d'autres termes, les paroles du schizophrène se conforment aux mêmes règles de rationalité et aux mêmes règles de logique que celles du locuteur non schizophrène avec lequel il s'entretient. Les auteurs expliquent donc certaines anomalies observées de la conversation en référence au niveau de l'analyse des règles d'usage du langage - ce qu'ils appellent parfois des «jeux de langage »-, c'est-à-dire des conventions rhétoriques et pragmatiques du discours ${ }^{15}$. Ce qui signifie que ce n'est pas la logique du discours schizophrénique, ni même sa sémantique, qui sont déficientes, mais que ce discours manque certaines conventions pragmatiques qui conduisent habituellement la conversation d'une signification ou d'un sujet à un autre. En effet, comme les auteurs le notent, généralement les anomalies de ces conversations sont fondées sur des termes ambigus ou des termes dont la morphologie est ambiguë. Par exemple, l'une des conversations analysées dévie sans transition de l'expression «être mort» prise au sens métaphorique (lorsqu'on perd à un jeu) à son sens littéral.

Du point de vue de l'interlocuteur, l'impression d'étrangeté dans ces conversations proviendrait de ces ruptures de l'interaction dialogique : nous pensions savoir de quoi traitait la conversation et soudain nous ne le savons plus et avons besoin de nous ajuster. Par conséquent, rendre compte de ces ruptures ne consiste pas simplement à appliquer le principe de charité, mais à combler les trous en restituant les liens conversationnels ou pragmatiques manquants ou implicites. Ce qui suggère que c'est l'usage des indicateurs habituels de transition dans la conversation qui serait défectueux. Selon Rebuschi et al., le dysfonctionnement de ces conversations relèverait donc d'une « inconsistance pragmatique » plutôt que d'un défaut de logicité ou de rationalité : les deux interlocuteurs ne font pas le même usage de certaines procédures dialogiques, mais ils en font bel et bien usage. Le discours schizophrénique ne manifeste pas un défaut de rationalité, mais témoigne plutôt d'un « déficit interactionnel ».

Or cette hypothèse se fonde sur les analyses du délire schizophrénique proposées par Sass à partir de la figure wittgensteinienne du solipsiste. Le solipsisme est alors compris comme une forme de scepticisme philosophique. Il est la conséquence logique d'une certaine posture philosophique qui enclot le sujet à l'intérieur de lui-même et du champ de ses expériences subjectives ${ }^{16}$. Il conduit à l'idée que «le monde est mon

15. M. Rebuschi et al., «Schizophrénie, logicité et perspective en première personne», art. cit., p. 134.

16. Dans les Méditations cartésiennes, Edmund Husserl résume très bien ce risque solipsiste d'une philosophie qu'il nomme (et revendique comme) une «égologie» et qui est « une 
monde » est qu'il n'y a rien de plus que je puisse en dire. Du point de vue solipsiste, je ne peux pas garantir l'existence réelle du monde extérieur, des autres (esprits) et de leur conscience - car ceux-ci pourraient tout aussi bien être des humains factices ${ }^{17}$ ou de simples objets de pensée; je devrais pouvoir être eux pour vérifier le contraire, ce qui est empiriquement impossible.

De manière semblable, remarque Sass, le monde du schizophrène peut revêtir cette texture étrange. Non pas que tout lui semblerait irréel ou que ce qui est irréel lui semblerait réel, comme le suggèrent les approches classiques de la schizophrénie ; mais plutôt, la réalité du réel serait pour lui constamment en question. Comme si le schizophrène était engagé dans une perpétuelle et récurrente remise en cause philosophique de l'existence du monde extérieur, prisonnier d'une forme d'épochè transcendantale. Néanmoins, loin d'être constamment sous l'emprise d'une illusion ou dans un état constant d'hallucination, le schizophrène (ou du moins certains schizophrènes), affirme Sass, serait dans un état d'hyper-intellectualisation, de réflexivité ou de "cérébralisation » excessive ${ }^{8}$. La schizophrénie serait l'incarnation existentielle des problèmes de la philosophie moderne et post-moderne.

Ainsi, comme semblait déjà le suggérer l'analogie wittgensteinienne, ce qui distinguerait le fou du philosophe tiendrait au fait que, pour le philosophe, la posture solipsiste n'est qu'une parenthèse, une posture théorique qui n'entache pas son rapport ordinaire ou quotidien au monde. Le philosophe peut tenir une position qui tend au solipsisme alors même que ses actions et la vie qu'il mène en dehors de ses méditations philosophiques sont incompatibles avec une telle position. Au contraire, le schizophrène, selon Sass, aborderait le monde depuis une posture solipsiste et éprouverait des difficultés à passer d'une posture réflexive à une posture ordinaire, où le réel s'impose tout autant que l'existence des autres esprits, où ceux-ci ne sont pas même en question ${ }^{19}$.

science absolument subjective [...] dont l'objet est, dans son être, indépendant de la décision sur le non-être ou l'être du monde. Plus encore, il semble que son premier et son unique objet soit et ne puisse être que mon ego transcendantal, celui du moi qui philosophe ». Ainsi, l'épochè transcendantale commence-t-elle par « une égologie pure [...] qui nous condamne, semble-t-il, à un solipsisme, même s'il est transcendantal. On ne peut absolument pas discerner comment, dans l'attitude de la réduction, d'autres ego [...] pourraient être établis comme étant destinés à être et à devenir ainsi le thème également légitime d'une égologie phénoménologique » (Paris, PUF, p. 74-75 - je souligne).

17. Un autre exemple de cette expérience de pensée est celui du passage bien connu de la deuxième des Méditations métaphysiques (op. cit., p. 75), où Descartes envisage la possibilité que les passants de sa rue ne soient que des automates: "[J]e dis que je vois les hommes mêmes. Or que vois-je sinon des chapeaux et des vêtements, sous lesquels pourraient se cacher des automates? ».

18. L. Sass, Les Paradoxes du délire, op. cit., p. 181.

19. Et où il ne s'agit donc même pas d'inférer d'une quelconque façon que les autres pensent (contrairement à ce que suggère une application abusive du principe de charité). 


\section{La rationalité du discours schizophrène}

Poursuivant en partie les hypothèses de Sass, les analyses de conversations avec des schizophrènes menées par Rebuschi et al., montrent que les interlocuteurs, lors de ces conversations, se trouvent confrontés à une situation dans laquelle certaines choses ordinairement prises pour argent comptant ne le sont pas. Les repères sur lesquels nous comptons habituellement pour nous accorder dans la conversation sont ébranlés. L'expérience solipsiste se déporte alors du côté de l'interlocuteur du schizophrène, ce dernier ne sachant plus si son point de vue ou son contexte de référence est le même que celui de son interlocuteur. Pour adapter une remarque de Wittgenstein, ce ne serait pas l'accord sur les définitions ou sur les significations qui ferait alors défaut, mais l'accord « dans le jugement» : ce ne seraient pas les méthodes de mesure ou les conventions qui ne seraient pas partagées, mais « une certaine constance dans le résultat des mesures ${ }^{20}$ ». Je développerai ce point un peu plus loin.

Pour le moment, admettons avec nos collègues de Nancy qu'il y a « de la rationalité » des deux côtés de l'interaction. Il faut donc également que nous puissions saisir le point de vue ou le contexte depuis lequel l'autre parle. En effet, le paradoxe du solipsisme réside en ceci que sa formulation même nécessite un point de référence depuis lequel nous pouvons le figurer. Il requiert donc une forme d'accord dont la possibilité même est remise en cause par ledit solipsisme. La possibilité d'adopter la posture du solipsiste est la condition même de sa formulation, et, pour ce qui nous concerne, la condition de possibilité d'une conversation avec la figure du schizophrène telle que représentée par nos auteurs.

D'un seul mouvement, le solipsisme perd donc sa dimension solipsiste. Dès lors, si la schizophrénie doit être comprise comme l'incarnation existentielle du solipsisme, elle ne peut être un authentique solipsisme. Car il n'y a pas d'authentique solipsisme : le solipsisme étant, comme l'a montré Wittgenstein, une impossibilité logique. (D'ailleurs, aucune philosophie encourant le risque du solipsisme - comme la phénoménologie de Husserl, par exemple - ne prétend qu'il puisse être autre chose qu'une attitude philosophique, distincte, donc, de l'attitude naturelle ou ordinaire). Le philosophe qui s'engage sur une telle voie doit donc être, en quelque sorte, de mauvaise foi. C'est sur ce point que l'analogie proposée par Sass se révèle difficile : il souhaite étendre le solipsisme à une façon de vivre, mais ce faisant, il anéantit la possibilité même de formuler le solipsisme. Si le solipsisme est une forme irrationnelle du doute ou son extension hors des limites de ce dont on peut raisonnablement douter, il doit reposer néanmoins nécessairement sur une certaine conception du doute raisonnable, rationnel. Ainsi, Descartes n'aurait pas pu faire appel à l'intuition de ses lecteurs si son doute hyperbolique n'était pas l'extension et l'exacerbation d'un doute

20. L. Wittgenstein, Recherches philosophiques, op. cit., § 242. 
ordinaire ; c'est-à-dire d'un doute régulé par des circonstances «normales », si normales qu'elles ne font l'objet d'aucune considération spécifique ${ }^{21}$. En d'autres termes, le solipsisme comme seuil d'une limite logique et comme extension abusive de figures ordinaires du doute ne peut se comprendre que sur la base du doute ordinaire.

Poussé dans ses retranchements, le solipsisme ne peut même pas être exprimé et ne peut relever que d'une sphère ou d'un langage privé, impartageable, intraduisible et incommunicable, c'est-à-dire ce qui n'est pas un langage du tout. Le paradoxe tient ici au fait que l'expression même du solipsisme prend pour point de référence une posture non-solipsiste. Ceci devrait donc être le cas également pour la figure du schizophrène : ce dernier ne peut interroger la réalité de ses perceptions que sur fond d'un concept relativement stable de "réalité ». La mise entre parenthèses ou la déstabilisation du réel ne peut se produire que par contraste avec une attitude « naturelle » consistant à saisir le réel comme tel ${ }^{22}$. Si tout est objet de doute, plus rien ne l'est. Le solipsisme s'annule lui-même. C'est de ce paradoxe, selon Sass, que le schizophrène est prisonnier, mais ce serait aussi à cette seule condition que nous pourrions le comprendre et lui nous comprendre. Le caractère paradoxal du solipsisme devient donc la condition de possibilité même de sa formulation et de la possibilité de se mettre dans la peau du solipsiste. C'est donc bien sur fond d'un commun qu'on peut comprendre la possibilité d'un point de vue dit « en première personne ».

Tout l'enjeu de cette comparaison entre le fou, le solipsiste et le philosophe tiendrait donc à la compréhension d'une certaine forme de «commun" irréductible qui nous rendrait capables de nous représenter minimalement le point de vue de celui qui profère des «non-sens ». Ceci nous ramène à la notion wittgensteinienne de forme de vie, qu'on ne peut véritablement caractériser positivement, mais seulement négativement ou localement, comme dans la remarque suivante :

Le chien croit que son maître est à la porte. Mais peut-il aussi croire que son maître viendra le lendemain ? - Et que ne peut-il pas faire ici ? - Comment le fais-je ? - Comment suis-je censé répondre à cela ? Est-ce que seuls peuvent espérer ceux qui peuvent parler? Seuls ceux qui maîtrisent l'usage du langage. Autrement dit, les phénomènes d'espoir sont des modes de cette forme de vie complexe. (Si un concept se rapporte à un aspect de l'écriture humaine, il ne s'applique pas aux êtres qui n'écrivent pas) ${ }^{23}$.

21. C'est ainsi, en effet, qu'il convient de comprendre «l'ordinaire » de Wittgenstein, comme ce qui va tellement de soi qu'il ne se voit pas, qu'il n'est pas dit, ni remarqué. C'est pourquoi voir et décrire l'ordinaire sont des tâches proprement philosophiques et ne consistent pas simplement à regarder autour de soi.

22. Voir la première des Méditations cartésiennes (op. cit.) de Husserl.

23. L. Wittgenstein, Recherches philosophiques, op. cit., II, i. 
À en croire ces remarques ${ }^{24}$, la forme de vie est ce qui est partagé par des êtres auxquels on peut appliquer certains concepts qui dépendent de cette même forme de vie. Malgré son caractère vague et général, cette notion de forme de vie a l'avantage de tendre vers l'idée qu'un individu schizophrène et un individu non-schizophrène ont quelque chose en commun (quoique difficile à décrire). Et c'est ce " commun » à partir duquel on peut envisager une compréhension possible. Pour le dire platement, la folie ne fait pas sortir de l'humain, de la forme de vie humaine. On ne peut se contenter de dire que ce qu'on ne comprend pas dans les pratiques et attitudes d'un autre, dans ce qu'on appelle parfois « le délire », est condamné à demeurer hors des limites du compréhensible.

Ce constat d'une forme de vie partagée rend possible une approche anthropologique de la folie, mais ne conduit pourtant pas, c'est ce que je souhaiterais montrer, à l'idée de rationalité partagée (sachant que le concept de rationalité est au moins aussi difficile que celui de forme de vie). Par " approche anthropologique », il faut entendre que les difficultés auxquelles nous confrontent les cas limites (comme la folie, ou une certaine attitude philosophique) ne sont pas extra-humaines mais se posent dans un contexte anthropologique. Le langage du schizophrène, entre autres, est bien le même que celui de son interlocuteur.

Dès lors, si nous ne touchons pas ici aux limites de l'humain (de sa forme de vie et même d'une certaine rationalité), il faut donc envisager une autre explication de ce que Rebuschi et al. appellent "déficit interactionnel ». Mais il demeure problématique de la faire reposer sur le principe de charité. Il est vrai que, lors de ces conversations, on voit surgir certaines difficultés qui n'émergent pas en contexte ordinaire. Ces difficultés ont trait à ce que les locuteurs signifient, veulent dire. Dans une conversation ordinaire (où l'ambiguïté n'aurait pas sa place), nous n'interprétons pas ce qui est dit. La compréhension mutuelle est spontanée et lorsque ce n'est pas le cas, nous employons (ou tentons d'employer) des moyens communs pour lever les éventuelles ambiguïtés.

En nous appuyant sur les analyses de Sass, nous pouvons suggérer que les difficultés qui émergent dans la relation avec le schizophrène proviennent d'un défaut de signification en contexte, alors même que les mêmes phrases ou les mêmes mots sont employés. Et ce défaut de signification proviendrait du fait que le contexte de référence des interlocuteurs n'est pas le même, bien que ceux-ci partagent un langage et une forme de vie. Il manquerait dès lors la possibilité d'un "accord dans le jugement», dans la mesure où le contexte de référence du schizophrène (pris dans la posture solipsiste) ne serait pas le même que celui de son interlocuteur. Le caractère d'étrangeté serait semblable à celui rencontré dans l'exemple de Wittgenstein par quiconque surprendrait une conversation de philosophes sans le savoir.

24. Voir également ibid., § 19, 23 et 241. 
La difficulté peut même aller, comme dans certains exemples de De la certitude, jusqu'à interroger mes "certitudes» les plus fondamentales. Il peut arriver un point où la seule chose que je puisse faire soit accepter les limites de ma compréhension :

Que pourrais-je répondre à des adultes d'une tribu qui croient que des personnes se rendent parfois sur la lune (peut-être est-ce ainsi qu'ils interprètent leurs rêves), bien qu'ils concèdent qu'on ne puisse y monter ou y voler par les moyens habituels ? ${ }^{25}$

Ainsi en va-t-il de certaines croyances fondamentales et de la croyance religieuse en particulier. Dans une certaine mesure, l'athée et le croyant doivent simplement admettre leurs divergences et reconnaître l'impossibilité de convaincre l'autre qu'il a raison ou tort sur ce point. Car ce type de croyance relève d'une dimension plus fondamentale de notre existence que d'autres. Il relève davantage, selon Wittgenstein, de la persuasion ou du dressage que de la justification :

N'est-ce pas là exactement la façon dont nous enseignons à un enfant à croire en Dieu, ou que Dieu n'existe pas, et il pourra, selon le cas, produire des justifications apparemment plausibles pour l'une ou pour l'autre croyance ${ }^{26}$ ?

Autrement dit, nous n'apprenons pas à croire en Dieu ou à ne pas y croire sur la base de fondements (épistémiques) ou de preuves qu'on nous présenterait, mais parce qu'on nous dit qu'il en est ainsi. C'est seulement une fois la conviction acquise que nous cherchons éventuellement à la justifier. Malgré ses limites, cette analogie avec la croyance religieuse témoigne de la variété des limites de la compréhension. Et ces limites ne confrontent pas nécessairement le rationnel à l'irrationnel, mais des certitudes ou des points de vue, non pas incommensurables, mais au moins incompatibles.

Faut-il cependant ressaisir ces variations anthropologiques et leurs rencontres sur la base du principe de charité et donc d'un certain concept de rationalité ? Et pouvons-nous maintenir dans ce cas l'idée d'une approche en première personne? Je voudrais conclure en suggérant que nous pouvons maintenir une certaine conception de la subjectivité, ou du point de vue en première personne, sans recourir au principe de charité.

25. L. Wittgenstein, De la certitude, op. cit., § 108.

26. Ibid., § 107. 


\section{Comprendre le délire}

En effet, l'idée que toute interaction (en particulier linguistique) se fonderait sur une certaine application du principe de charité est tout à fait discutable d'un point de vue wittgensteinien. Car cette idée repose sur la supposition que toute interaction relèverait de l'interprétation. Autrement dit, mon accès aux pensées d'autrui ne se ferait que sur la base d'une interprétation charitable de celles-ci : je suppose qu'autrui est au moins aussi rationnel que moi. Mais, cette hypothèse repose sur un subjectivisme méthodologique, sur l'idée que c'est depuis ma propre rationalité que j'accède à celle d'autrui. Cependant, si c'était le cas, on pourrait se demander si cela a même un sens de parler de rationalité du discours. En réalité, si toute interaction était interprétation, il n'y aurait plus de critère à l'interprétation ; c'est justement le problème de Quine. Il peut néanmoins sembler plus plausible de n'invoquer la nécessité d'une interprétation que là où précisément il $\mathrm{y}$ a de l'ambiguïté, là où il $\mathrm{y}$ a un doute interprétatif. Ce qui est le cas dans la confrontation avec le délire. C'est pourquoi le principe de charité peut sembler si adapté au cas de la parole schizophrénique. Mais dès lors on pourra se demander s'il ne s'agit pas de plaquer sur cette parole des normes de rationalité qui lui sont externes - et retrouver du même coup une perspective en troisième personne plutôt qu'en première personne. Ainsi la possibilité d'une perspective en première personne sur le discours schizophrène, au sens envisagé par Rebuschi et al., s'avère problématique.

C'est pourquoi je voudrais suggérer des pistes pour l'envisager autrement, notamment à partir de l'idée que la déficience des conversations n'est pas seulement interactionnelle - au sens où elle devrait être réparée par l'explicitation de liens rationnels manquants - mais doit tenir compte des perspectives individuelles prises sur le contexte ou l'occasion de l'interaction. L'idée d'un point de vue en première personne ne supposerait pas ici la transposition d'une rationalité sur une autre, mais une communauté de forme de vie suffisante pour pouvoir envisager (jusqu'à un certain point seulement) le point de vue de l'autre. Il serait dès lors nécessaire de pouvoir restituer les points de vue de chacun des interlocuteurs dans le cadre de l'interaction. Il ne suffirait pas simplement à l'un (tenant de la rationalité) d'interpréter le discours de l'autre, mais il faudrait également ressaisir la façon dont l'autre saisit le discours de l'un.

Pour cela, il semble insuffisant d'expliquer les décrochages de la conversation à partir de simples défauts d'usages de procédure dialogique comme si tout se passait en contexte strictement linguistique. Ou du moins, ce concept d'usage aurait besoin d'être réélaboré : car s'il a à voir avec une difficulté relative au contexte de référence sur lequel s'appuie chacun des interlocuteurs, alors il ne peut pas se réduire à la simple application de règles pragmatiques du discours prévues d'avance. Il faut, comme le dit Sass, se rapporter à des éléments extérieurs au langage - et 
peut-être même une certaine Weltbild ou Weltanschauung ${ }^{27}$, qui n'est pas nécessairement quelque chose dont nous pouvons rendre compte de manière explicite ou exhaustive, mais qui ne renvoie pas non plus à une expérience ineffable ou privée.

Nous pourrions admettre que la parole du schizophrène n'est pas paraconsistante: les contradictions apparentes n'y sont pas de véritables contradictions. Mais ce qui ferait défaut pour la comprendre ne se limiterait pas à des éléments pragmatiques ou sémantiques, mais à des éléments contextuels relatifs à des disparités de Weltanschauung, de «toile de fond » de la parole (pour parler comme Wittgenstein).

Il nous manquerait ainsi des éléments pour saisir ce que signifient les individus, en un sens de « signifier » qui ne se limite pas à la question de la traduction d'une langue dans une autre, mais qui s'étend à la question du contexte et du point de départ de l'énonciation. En d'autres termes, ce n'est pas que les mots du schizophrène auraient un sens inédit (ou privé), mais il nous manquerait des éléments contextuels au sens large du point de vue depuis lequel la parole s'exprime. Nous aurions affaire à un défaut de ce que Travis (2008) nomme «sensibilité à l'occasion » et qui fait écho au fameux passage des Recherches philosophiques de Wittgenstein mentionné plus haut et que nous pouvons désormais citer en entier :

Pour que le langage soit un moyen de communication, il doit y avoir non seulement accord dans les définitions mais également (aussi étrange que cela puisse paraître) accord dans les jugements. Cela semble abolir la logique, mais ce n'est pas le cas. - C'est une chose de décrire les méthodes pour mesurer et une autre d'obtenir et d'établir les résultats de la mesure. Mais ce que nous appelons «Mesurer» est en partie déterminé par une certaine régularité dans les résultats de la mesure ${ }^{28}$.

L' ' accord dans les jugements » ne porte pas simplement sur la logique interne au langage, mais aussi sur ce que nous signifions en une occasion donnée en disant. Pour cela il ne suffit pas de partager les méthodes de mesure, mais aussi « une certaine régularité dans les résultats de la mesure ». Et ceci ne peut s'accomplir qu'à travers ce que Travis nomme «le paroissial », à savoir une forme de compréhension partagée de la situation et de ce dont il est question dans le discours. L'incompréhension elle-même exige cette dimension du « commun » dans l'interaction.

De ce point de vue, les ruptures dans les conversations avec le schizophrène, observées par Rebuschi et al., pourraient s'expliquer davantage par un défaut lié au « sens du paroissial », qui se rapporte à ce que

27. Décrite par Wittgenstein de la manière suivante (De la certitude, op. cit., § 94) : «Mon image du monde, je ne l'ai pas parce que je me suis convaincu de sa justesse ; ou parce que je suis convaincu de sa justesse. Elle est la toile de fond dont j'ai hérité et sur laquelle je distingue le vrai du faux ».

28. L. Wittgenstein, Recherches philosophiques, op. cit., § 242. 
les individus " ont en tête » (comme on dit) au cours de la conversation (et qui, encore une fois, n'a nul besoin d'être par principe privé, mais qui peut être difficile d'accès dans certains contextes) :

J'appellerai «paroissiale» toute forme, ou figure, de la pensée d'un être, qui n'est pas simplement exigée par le fait d'être un penseur en tant que tel; de sorte qu'il se peut qu'il y ait des penseurs dont la pensée n'aurait pas ce trait j'appellerai donc paroissiale toute forme de pensée pour laquelle il pourrait y en avoir d'autres. Ainsi, n'importe quel trait de ces généralités sous lesquelles nous pouvons (are equipped to) envisager les choses (les présenter à nousmêmes), susceptible d'être absent du stock de généralités à partir desquelles un autre type de penseur peut envisager les choses, sera paroissial en ce sens ${ }^{29}$.

Le caractère «paroissial» d'une pensée tient donc au rapport étroit qu'elle entretient avec un penseur ou des penseurs donnés en une occasion particulière. Cependant, cette pensée, bien qu'elle soit pour ainsi dire relative ou sensible à une occasion donnée de la dire ou de la penser, doit être ou pouvoir être exprimée dans les mots du langage et n'est donc pas, en ce sens, privée ou incommunicable. Ce que Travis nomme " sensibilité à l'occasion » a précisément à voir avec la capacité des mots et des phrases à changer de signification selon la variété des occasions de leur emploi. Le sens du paroissial est alors précisément la capacité du locuteur à signifier ce qu'il veut dire en une occasion donnée (et, le cas échéant, à comprendre ce qui est dit) et même en vertu de certaines circonstances particulières. Si les ruptures de conversation qui nous intéressent peuvent alors se comprendre par un défaut de ce sens du paroissial, c'est donc «l'accord dans le jugement » qui est en cause. Tout se passe comme si, à un moment donné, les interlocuteurs cessaient de mener la même conversation, comme s'il se produisait un glissement imperceptible de contexte. Mais alors ce glissement de contexte concernerait les deux interlocuteurs : il se produit, pour ainsi dire dans les deux sens, et c'est la compréhension mutuelle qui est altérée. Ce n'est pas seulement la possibilité de comprendre qui est altérée, mais celle d'être compris par son interlocuteur.

Cette hypothèse n'a rien d'une solution si notre problème est de donner un sens à la parole du fou. D'un autre côté, s'il existait une telle solution, nous ne pourrions plus qualifier cette parole de « folle » ou de « délirante ». L'intérêt de l'analogie entre le fou, le solipsiste et le philosophe est de nous mettre en présence de cas où précisément nos concepts ordinaires sont mis en défaut ; ils sont comme décrochés de leurs usages, et c'est cela qui donne au discours son apparente étrangeté.

Or si nous avons avancé sur un point, au terme de cette analyse, c'est celui qui consiste à voir que ces cas limites, tout en déstabilisant nos concepts, les mobilisent bel et bien. Et c'est précisément parce qu'ils les mobilisent qu'on ne peut pas admettre qu'ils relèveraient du strict non-sens,

29. Ch. Travis, Objectivity and the Parochial, Oxford, Oxford University Press, 2010, p. 1-2. 
au sens des lectures dites «austères» du non-sens ${ }^{30}$, qui excluent radicalement le non-sens du langage et du dire. En effet, rejeter le discours métaphysique ou le discours délirant du côté de la pure et simple absence d'un dire, c'est nier la dimension anthropologique de ces discours, c'est les détacher d'un vouloir dire et d'une action (de dire ou d'essayer de dire) qui a pourtant bien lieu. C'est faire du discours une entité idéale, qu'on pourrait extraire des pratiques et analyser dans sa pureté même de discours.

Ce que Sass et Rebuschi et al. ont bien saisi, dans leur volonté de restituer une perspective "en première personne", c'est la nécessité de reconnaître qu'on a bien affaire à du dire (au sens de l'action de dire) et pas simplement à une parodie de langage. Ils ont donc bien vu la nécessité d'un point de vue anthropologique sur ce dire, au sens où, en ce qu'il est un dire, il ne nous fait pas sortir de l'humain, ni même du langage, en ce qu'il constitue une part de ce que j'ai maladroitement appelé ici le «commun ». Ce qu'ils ont échoué à saisir dans toute sa radicalité, c'est la dimension de limite à laquelle nous confrontent ces cas limites. En effet, une chose est d'y reconnaître un dire, une autre est de vouloir les extraire de leur situation limite en en rendant raison et en les raccrochant à une norme à laquelle ils sont certes apparentés, mais de laquelle justement ils se démarquent. Autrement dit, il faut accepter que la limite à laquelle nous nous trouvons confrontée est bel est bien insurmontable dans la mesure où elle déstabilise « la toile de fond» du discours lui-même, ce qui va sans dire. Mais cela ne saurait nous empêcher d'essayer de saisir une certaine forme d'expérience ou de vérité grammaticale dans la parole du fou, du solipsiste et du philosophe. Pour cela, peut-être faut-il procéder à la manière de Wittgenstein lorsque, confronté aux grandes questions métaphysiques, il cherche dans la grammaire du langage la source de ces perplexités. En effet, si le non-sens métaphysique recèle des vérités grammaticales selon Wittgenstein, c'est au sens où il transforme en expériences existentielles des possibilités de la grammaire, où raisonner à partir du possible ouvre des perspectives vertigineuses (comme celle du solipsisme). De la même manière, il nous faut reconnaitre que le discours « délirant », tout en n'étant pas « ordinaire », est l'expression d'une expérience possible qui " nous » est étrangère, sans jamais rien concéder à son caractère «limite", c'est-à-dire au fait que ce discours nous place face à notre propre langage dans une situation d'inconfort et d'incompréhension. Reconnaître à la parole délirante sa dimension de parole, ce n'est pas nécessairement lui conférer un sens ou la traduire en une parole sensée. Mais c'est la reconnaître comme l'expression d'une certaine expérience non partagée (voire même comme une tentative de la dire), c'est reconnaître qu'elle n'est pas que du langage mais aussi et surtout la parole d'un individu qui partage et a appris le langage. Or reconnaître le discours délirant (au même titre que celui du métaphysicien) comme un dire (ou au moins une tentative de dire), c'est se donner la

30. Voir, par exemple, C. Diamond, The Realistic Spirit, Cambridge MA, The MIT Press, 1991 ; A. Crary et R. Read (éds.), The New Wittgenstein, Londres, Routledge, 2000. 
possibilité de chercher ce que celui qui le prononce a voulu dire, que ce soit dans la grammaire de notre langage ou dans la conversation elle-même. Il ne s'agit pas de croire naïvement qu'il suffirait de poursuivre la conversation pour se comprendre, car parfois nous atteignons les limites de ce que nous pouvons comprendre; d'autres fois encore, la poursuite de la conversation est impossible car le fossé entre les interlocuteurs est trop grand. Il s'agit néanmoins de reconnaître la possibilité d'un vouloir dire dont la saisie ne s'épuise pas dans une reconstitution externalisée des éventuelles lacunes d'un discours ou d'une conversation, mais qui exige, comme d'ordinaire, que l'on s'intéresse à ce que le locuteur lui-même pourrait bien avoir à expliciter (dans la mesure du possible) des lacunes de son propre discours. 